\title{
Internet of Things and Blockchain-based framework for Coronavirus (Covid-19) Disease
}

Tanweer Alam

Faculty of Computer and Information Systems

Islamic University of Madinah, Saudi Arabia

Email: tanweer03@iu.edu.sa

\begin{abstract}
The COVID-19 is an exponentially growing disease that has intentioned nations to use technologies to detect the coronavirus infection. Several nations are working greatly to fight against COVID-19. Many nations have been using a range of devices to combat the pandemic, seeking information about growth, monitoring as well as the leaking the confidential information of the residents. This research aims to assist infected people online using the Internet of Things (IoT) and Blockchain technologies through smart devices. IoT-based healthcare devices gather useful information, provide additional insight through symptoms and behaviors, allow remote monitoring, and simply give people better self - determination and healthcare. Blockchain allows the secure transfer of patient health information, regulates the medical distribution network. A four-layer architecture is proposed using IoT and Blockchain to detect and prevent individuals to be COVID 19. This research provides a framework for patients with COVID-19 infectious disease and recognizes health issues and diagnoses online. Smart devices such as smartphones can install any mobile apps such as Aarogya Setu, Tawakkalna, and so on. These applications can track COVID-19 patients properly. The installation of mobile apps on smart devices focuses to reduce the time and cost and increase the performance of the infectious patient's condition. A four-layer architecture is proposed using IoT and Blockchain technologies. Many research works focus on investigating, analyzing, and highlighting the affected individuals through guiding the COVID-19 infection. Eventually, various mobile apps are recognized and addressed in this paper.
\end{abstract}

Keywords: COVID-19, IoT, Blockchain, Contact Tracing, Mobile Applications.

\section{Introduction}

The pneumonia infection triggered through coronavirus happened in Wuhan china, in Dec 2019 and has spread like wildfire nearby Wuhan areas [1]. That bacterium with this epidemic has been simply referred to as COVID 19. The generation of the vaccines as well as experimentation on COVID-19 treatments are ongoing, however, it would take several months, approximately the end of 2020. Besides that, pressures rise in the future on the worldwide healthcare system. There are two different forms of this stress. Firstly, the potentially overwhelming challenge of diseases which concerns the quality of healthcare structure. Secondly, the negative impacts on healthcare professionals, such as the high risk. The antibody response is responsible for preventing and eliminating COVID 19 infections, furthermore, self-adjusted antibody cells could contribute to immune responses and disordered respiratory system [2]. 


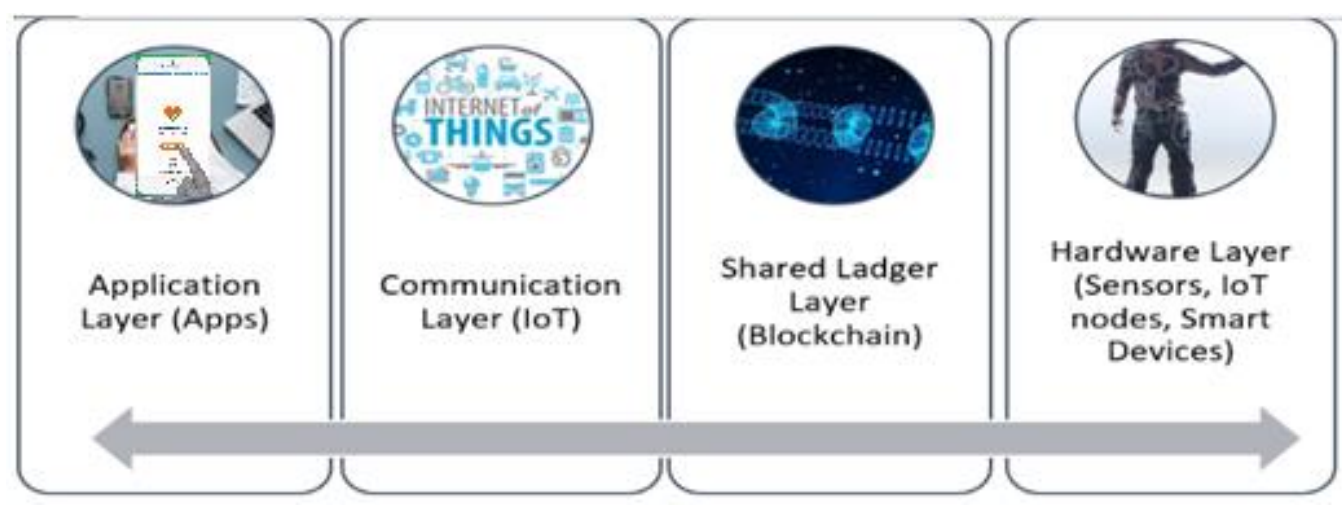

Figure 1. Four Layer Framework

Smartphones are devices that can be used to fill the gap among people and organizations, and they might have an effectively major role to play in resolving a further step of COVID 19 reaction. Touch and distance monitoring applications are becoming famous throughout Asian countries. The strategy differs across various cultures, including a tendency generally preferring such devices in favor of healthcare professionals. Figure 1 shows the fourlayer architecture of the proposed framework [3].

New technologies have enlisted the help to diagnose many infected, recognized the areas where COVID 19 is spreading, and find information dynamically [4]. Although an information security problem would be at risk, almost all of the applications developers nowadays are attempting to bring in active measures to protect the privacy of users [5].

Various mobile applications are being developed or suggested in some counties and states, with funding. Many methodologies have been proposed for constructing interaction detection software or apps [6]. The COVID-19 apps are the application development technologies that are using electronic monitoring to help to track people in reaction to the COVID-19 disease outbreak [7], it also identifies people who would have been in contact with the infected one. World Health Organization (WHO) is continuously changing the COVID-19 database through the ArcGIS GeoEvent service to send changes several times every day [8]. Contacts tracking apps are the relevant means of controlling infections, however, new cases continue to increase, limitations making appropriate controlling of transferring of diseases more complicated [9].

Volunteer, as well as occasional mobile applications to help track contacts, are essential methods to meet a potential challenging problem. Numerous researchers are working on simultaneous programs, and alternative scenarios have been explored by healthcare professionals throughout various nations. This becomes vital that healthcare officials, physicians and employees members everyday interaction, tracking infectious people and actively engaged in the activity to ensure which applications perform in line to peoples highest quality understanding of COVID-19 diagnosis, so the mobile apps have been built to accommodate conventional contact tracking initiatives [10].

The COVID-19 mobile treatment can strengthen the effect of self-isolation, information gathering, and monitoring of "hot spots" infections [11]. Throughout fact, we think that the public is involved with using smartphone-based technology to monitor and treat certain diseases - so there may be going to be a takeover. At-home monitoring would unlock money or avoid COVID-19 for reducing accessibility to hospitals, which would raise complications for a certain situation [12]. 


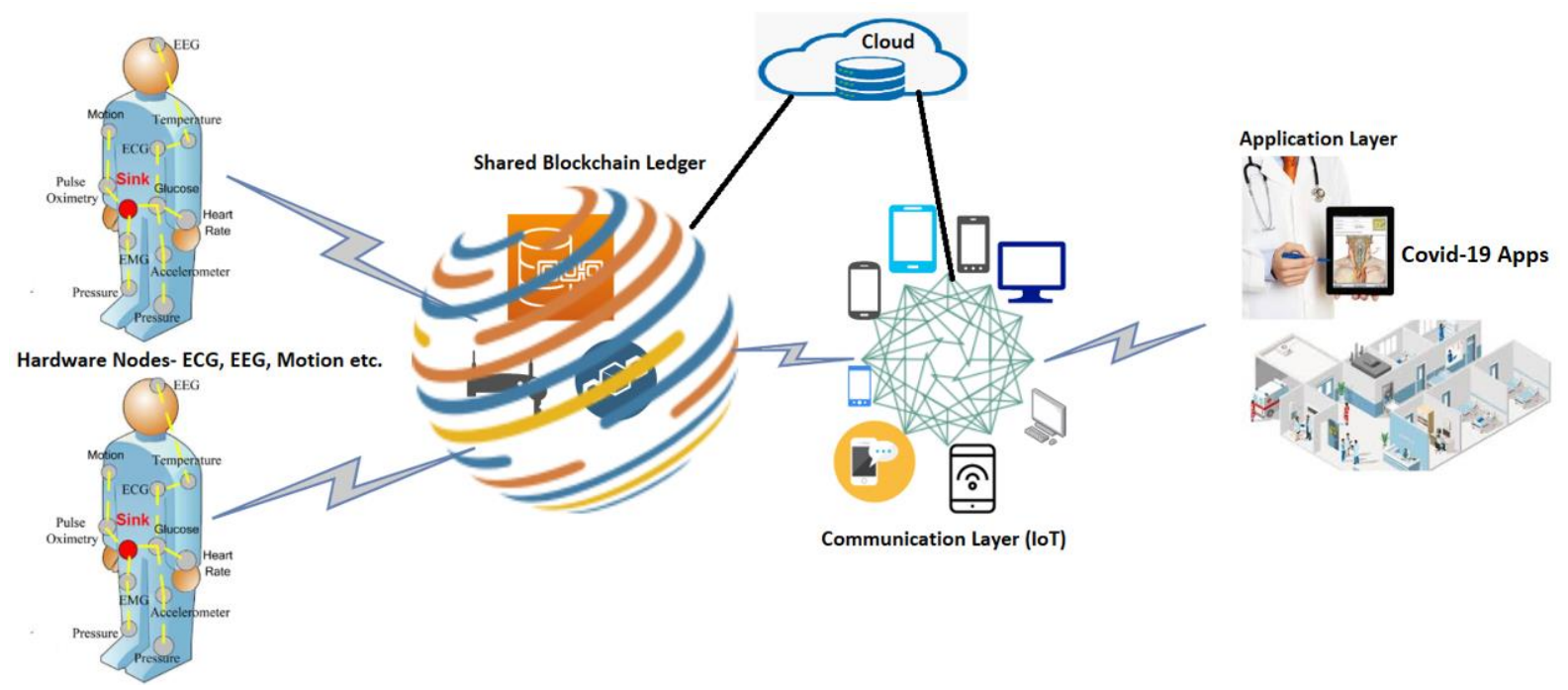

Figure 2: IoT and Blockchain-based Framework for Healthcare

Throughout the paper, the blockchain-based method is built to improve the reliability of physiological information and to improve transmitting data efficiency using the shared system to protect data records from attacker node manipulation threats [13]. Additionally, smart contracts are proposed to predictive analytics of sensitive personal real-time information. Smart contracts should operate as trusted parties to support two or more parties to discuss information in a secure manner [14].

\section{Smart Apps to detect the coronavirus}

The Coronavirus apps are smartphone programs that used Electronic interaction tracking for responding to the coronavirus epidemic, for example, the identification processing of individuals interaction who might be in a touch of active infection. Various mobile apps are developing in some counties, through approved government funding. Many other approaches are proposed for developing contact detection applications. Like the COVID-19 disease outbreak panics, research scientists are now hurrying all-around to create contact tracing devices, programs, and applications for recognizing and alerting everyone who falls into touch with infected people. Some applications are developed by different nations. Some of them are described as bellow.

\subsection{Aarogya Setu}

This app records positioning information that needs a regular connection to the Mobile device, that is intrusive from a privacy and security perspective. It can do this, as well as to facilitate legal compliance guidelines, etc. [15]

\subsection{Kwarantanna domowa}

Along with other issues, this app gives the possibility of investigating regional health insurance institutes, that in reasonable situations would provide treatments as well as souvenirs. People could also rapidly notify a staff member. App users often have speedy access to appropriate information-helpful during quarantine and a counseling service communication strategy besides people. Designers also enhanced it a few days earlier with a self-monitoring configuration for medical care. This application officially developed by Poland [16]. 


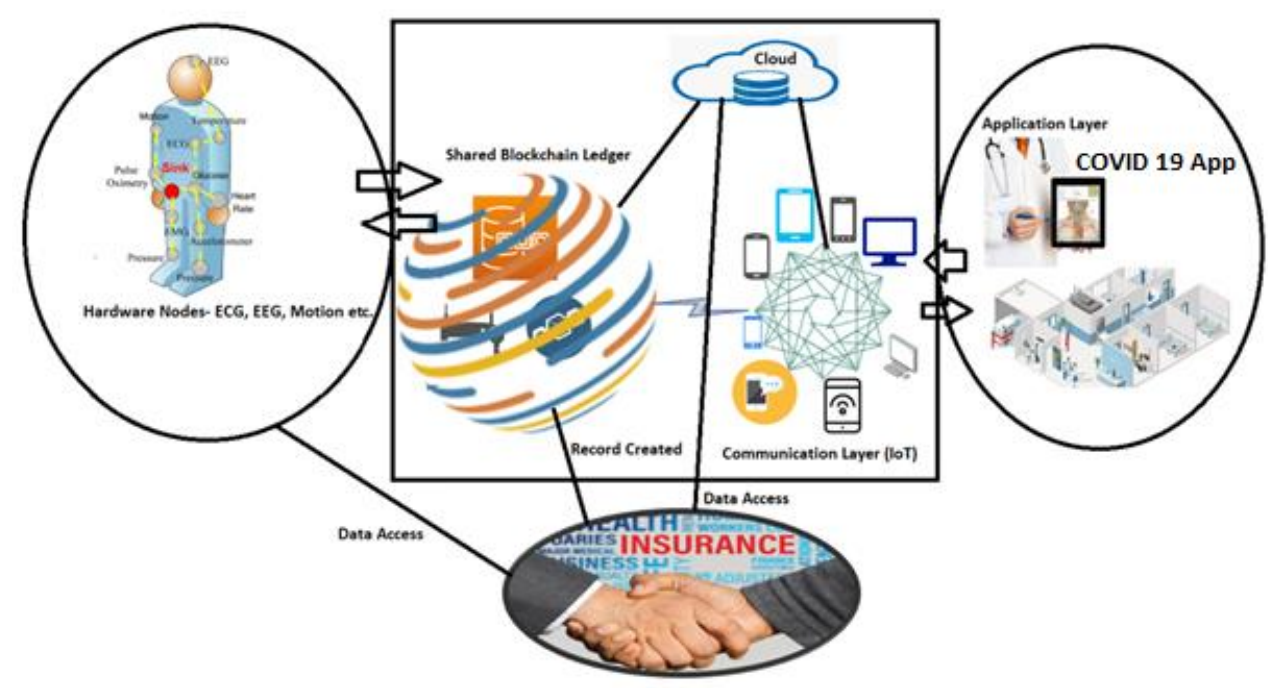

Figure 3. Framework connected with Insurance in real-time

\subsection{Tawakkalna (Covid-19 KSA)}

This application's goal is to track people's movements throughout KSA. This includes the movement's permission and every patient's health issue. This is a simulator of the China project. It is a color-based coding program representing the individual's condition. The green color indicates clean and allowed to travel. The yellow color represents the offender, and not permitted to travel. The red means affected as well as restricted from traveling. For checking the efficacy of this app, it is undergoing and in the trial version. A way to reach the community is to give everyone SMS randomly to give facilities to everyone. Saudi Arabia's ministry of health is supporting this app [17].

\subsection{TraceTogether}

It is designed to allow community-driven touch tracking to assist ongoing regional action to tackle the COVID-19 pandemic. This app was launched by the Singapore government that uses the customized Blue-trace standard to enable touch tracking digitally [18]. It was created and published on March 20, 2020, by Public information technology. It has been accessed by 17 percent of the total population since the launching of the application, which means more than $2,100,000$ users.

\subsection{LetsBeatCOVID}

It is developed to give individuals to perform a brief discussion regarding fitness including COVID-19 risk in efforts to save more lives of people [19]. This was published by MedShr, a medical application utilized by more than a million physicians. Citizens are requested to review it out a brief online questionnaire regarding himself, but they're also required to link in their relatives' details.

\subsection{CovidWatch}

This app was created through a partnership with Stanford University, the true courage people to support himself and the neighborhoods while giving up confidentiality. This utilizes Bluetooth signals to identify individuals whenever they are close to one another, or secretly warns people when they're in touch with infected people. The distinctive aspect of the 
application is that every partner, like the authorities, cannot monitor who has been reported in which. With private information-preserving, collaborative Bluetooth touch tracking, this was one of the first applications to launch an open-source specification [20].

\subsection{Other Apps}

Several other applications are developed such as HaMagen, Covid-19 Tracker, Corona DataSpende, NHS, COVID Symptom Tracker, etc. and a lot of mobile applications are under development stages.

\section{Methodology}

The present incident caused everyone to rethink several of common views and attitudes. All expansion of COVID-19 motivated everyone to prevent something but placed the security of individual fitness at the center of attention. Everyone now beginning their routines again, It has been thinking how to do anything securely. Dentistry hospitals are regarded as another of the conditions much more at the possibility of viral infection and pathogens infection and became a significant target technology for the expansion of any diseases like COVID19 disease.

Throughout the current smart technology world, position monitoring is a really difficult and important topic. Users could even activate the optimistic request or obtain one somewhere else in the area of the network, at a certain moment and any moment [21]. That platform connects the growing numbers of Cellular access points [22]. Furthermore, position administration and handoff control are recognized as cellular networks [23]. Location tracking allows the cellular connection to locate the telephone in between two neighboring communications [24]. Handoff leadership promoting cellular in locations outside the residence or even while traveling [25].

Blockchain technologies have been one of the innovation adoptions in recent years that might have selected by the world seriously. A blockchain is nothing but a distributed ledger that continues to keep processes and incident records traveling on all over the framework. Another very significant thing of a blockchain is that no one can alter after a piece of information is added to the distributed network. The data saved on the blockchain is in reality safe. This was mandatory to make significant adjustments for all subsequent layers after this one across the interchange for anybody to implement the switch to a module. The IoT is becoming both a familiar and evolving phenomenon that allows the use of sensors for online interaction.

A database has become a key basic foundation of healthcare services. Medical data computerization will have created great possibilities in terms of data transmitting and patient monitoring. Medical information has grown rapidly, with more and more knowledge-producing outlets, many within health organizations. Protection at knowledge is a rising issue. The solution uses blockchain technology at the structural stage of the network, getting transmitted by default, behaving as a peer-to-peer system aims to enable the different entities, relating to various providers or organizations which could store, create and/or modify mobile healthcare data. Figure 2 shows the IoT and Blockchain-based Framework for Healthcare system.

The hash function of blockchain technology has made it possible to authenticate users, the validation of blockchain-based post legitimacy is carried out by checking the hash keys. Consumers can check the authenticity of the data that does not want to recalculate everything 
the hashing to authenticate the transaction details and may ask for Merkle's proof, it involves combining the branches' left and right key and checking the result against the member of families.

Hardware Layer is composed of sensors and/or devices gathering and transmitting data to the upper section. Since sensors collect patient clinical information, the sensor node would have been validated before it joins the system. Its result should only be found important when it has been checked successfully. Devices and sensors collect data and will be processed in the blockchain network. A distributed database gives excellent functionality and performance, especially as opposed to heterogeneous networks which would generate traffic congestion at the center location.

Various communication methods may be used in the transmission medium to facilitate the exchange of information between devices connected to the internet. That data is captured by transmitting it to mobile devices used by consumers as well as healthcare professionals. Agreement among patients and healthcare services professionals must be developed so that user data might be shared with confidentiality. Thus, the platform generated with smart devices can be built as a peer-to-peer network.

The application layer contains the cooperation of several applications/services to accomplish collective behavior. Besides example, an implementation could be designed by a medical practitioner who's been requesting the information of the senses obtained from the devices of the service user. Clients will approve the data before it is sent to the network, which verifies the signature to ensure the security of data. If the authentication is reliable would be agreed upon appropriate diagnosing suggestion information supplied; that perhaps the information will be dismissed after all. Figure 3 shows the IoT and blockchain-based framework for a healthcare system with insurance.

This healthcare system is becoming an information-intensive system that requires vast quantities of regularly generated, analyzed, and transmitted information. Throughout organizational-centric patient information, even so, patient data is commonly separated, contributing to the incoherence of impacts differing from ineffective cohesiveness of provider to importance of necessary data through most of the emergency situations. A distributed computing tracks each activity approved, and it has been replicated among authorized users. It is a method of a shared database - transparency is assured by a shared database with authorized, checked, protected exchange of data.

\section{Discussion}

Coronavirus is the big family of viruses that affect humans from a simple cold to further serious conditions. The novel coronavirus is now a new disease unidentified among people before. Coronavirus is highly pathogenic which means that they might be spread among human beings and animals. Severe symptoms, sore throat, cough, shortness of breath, and fever are familiar examples of diseases. With more urgent conditions, pneumonia, extreme respiratory distress condition, kidney disease as well as deaths may result from infections. The set of circumstances indicates that now the coronavirus might've been transferred by the symptomless transporter [26].

Normal guidelines for preventing transmission of the disease involve daily washing hands, face and noses shielding while cough or sneeze, careful preparing meals of meat products. 
Misunderstanding and myths about the infection frequently occur while on an outbreak. Individuals would get a range of interesting news, friends, family, social networking sites, institutions, or even other types of data. Several of these news agencies could provide contradictory reports. The WHO motivates all nations to implement their monitoring of infectious diseases, to continually evaluate such a suspicious behavior of situations of infection, and even to inform WHO of every person infected scenario of coronavirus infectious disease.

Mobile-based testing was done to identify HIV, malaria, tuberculosis, and other toxins in the meals. Research has also been ongoing to be using mobile to identify COVID-19 but there are numerous concerns regarding the simplicity and effectiveness that use technologies in the direction.

Blockchain technologies now have the potential to change healthcare systems. This does offer an incentive to promote appropriate market approaches in the health sector, though. Reliable sharing of patient data may lead not only to things becoming conducted appropriately, and also to the application of new strategies. Successfully giving people the authority of their health records will be some of the key major effects. Blockchain technology will theoretically require permission and access to medical data to be managed by patients whenever they see worthy.

\section{Conclusion}

The WHO brings closely researchers from around the globe including government officials to promote the cycle of research and innovation and create international policies to control the transmission of the coronavirus epidemic and increase support for everyone infected. Testing COVID-19 might be possible through mobile in the future. Testing HIV, malaria and Tuberculosis are already being done through mobile. People would be used to identify and check substances, antibodies produced against viruses. This could appear far-fetched however the mobile can be used to diagnose patient conditions. Smartphones could be converted into instruments to easily detect a range of disease-causing agents like viruses, contaminants, and diseases. This framework provides a range of flexibility goods and services. The presence in remote diagnostics and monitoring, patient data, medical informatics, sensitive data health, public health information gathering, healthcare professionals, and information technology. The number of influences of health technology differs significantly depending on the sector. Its performance of mobile healthcare services is dependent on the level of development and the functionality of each service industry.

\section{References}

[1] Rodriguez-Morales, Alfonso J., D. Katterine Bonilla-Aldana, Ruchi Tiwari, Ranjit Sah, Ali A. Rabaan, and Kuldeep Dhama. "COVID-19, an emerging coronavirus infection: current scenario and recent developments-an overview." J Pure Appl Microbiol 14 (2020): 6150 .

[2] Li, Geng, Yaohua Fan, Yanni Lai, Tiantian Han, Zonghui Li, Peiwen Zhou, Pan Pan et al. "Coronavirus infections and immune responses." Journal of medical virology 92, no. 4 (2020): 424-432.

[3] Alam, Tanweer. "mHealth Communication Framework using blockchain and IoT Technologies." International Journal of Scientific \& Technology Research 9, no. 6 (2020). 
[4] Singhal, Tanu. "A review of coronavirus disease-2019 (COVID-19)." The Indian Journal of Pediatrics (2020): 1-6.

[5] Jiang, Shibo, Shuai Xia, Tianlei Ying, and Lu Lu. "A novel coronavirus (2019-nCoV) causing pneumonia-associated respiratory syndrome." Cellular \& molecular immunology 17, no. 5 (2020): 554-554.

[6] Mehta, Puja, Daniel F. McAuley, Michael Brown, Emilie Sanchez, Rachel S. Tattersall, Jessica J. Manson, and HLH Across Speciality Collaboration. "COVID-19: consider cytokine storm syndromes and immunosuppression." Lancet (London, England) 395, no. 10229 (2020): 1033.

[7] Kannan, S., P. Shaik Syed Ali, A. Sheeza, and K. Hemalatha. "COVID-19 (Novel Coronavirus 2019)-recent trends." Eur. Rev. Med. Pharmacol. Sci 24, no. 4 (2020): 20062011.

[8] Udugama, Buddhisha, Pranav Kadhiresan, Hannah N. Kozlowski, Ayden Malekjahani, Matthew Osborne, Vanessa YC Li, Hongmin Chen, Samira Mubareka, Jonathan B. Gubbay, and Warren CW Chan. "Diagnosing COVID-19: the disease and tools for detection." ACS nano 14, no. 4 (2020): 3822-3835.

[9] Iyengar, Karthikeyan, Gaurav K. Upadhyaya, Raju Vaishya, and Vijay Jain. "COVID-19 and applications of smartphone technology in the current pandemic." Diabetes \& Metabolic Syndrome: Clinical Research \& Reviews (2020).

[10] Kumar, Aishwarya, Puneet Kumar Gupta, and Ankita Srivastava. "A review of modern technologies for tackling COVID-19 pandemic." Diabetes \& Metabolic Syndrome: Clinical Research \& Reviews (2020).

[11] Yasaka, Tyler M., Brandon M. Lehrich, and Ronald Sahyouni. "Peer-to-Peer contact tracing: development of a privacy-preserving smartphone app." JMIR mHealth and uHealth 8, no. 4 (2020): e18936.

[12] Singh, R.P., Javaid, M., Haleem, A. and Suman, R., 2020. Internet of things (IoT) applications to fight against COVID-19 pandemic. Diabetes \& Metabolic Syndrome: Clinical Research \& Reviews.

[13] Tanweer Alam, "IoT-Fog: A Communication Framework using Blockchain in the Internet of Things", International Journal of Recent Technology and Engineering (IJRTE), Volume-7, Issue-6, 2019.

[14] Tanweer Alam, Mohamed Benaida, "Blockchain, Fog and IoT Integrated Framework: Review, Architecture and Evaluation”, Technology Reports of Kansai University, Volume - 62 , Issue 02, 2020.

[15] App, Aarogya Setu Mobile. "MyGov. in.(2020)."

[16] Długosz, Piotr. "Raport z badań:,Krakowscy studenci w sytuacji zagrożenia pandemią koronawirusa"." (2020).

[17] Perveen, Shagufta, Raha Orfali, Shafiq Azam, Hanan Y. Aati, Khulud Bukhari, Sara I. Bukhari, and Areej Al-Taweel. "Coronavirus nCOVID-19: A Pandemic Disease and the Saudi precautions." Saudi Pharmaceutical Journal (2020).

[18] TraceTogether, Team. "How does TraceTogether work?."

[19] Rosenkrantz, Leah, Nadine Schuurman, Nathaniel Bell, and Ofer Amram. "The need for GIScience in mapping COVID-19." Health \& Place (2020): 102389.

[20] Watch, Covid. "Covid Watch." last access 7 (2020). 
[21] Alam, Tanweer. (2018) "A reliable framework for communication in internet of smart devices using IEEE 802.15.4." ARPN Journal of Engineering and Applied Sciences 13(10), 3378-3387.

[22] Alam, Tanweer, and Mohammed Aljohani. "Design and implementation of an Ad Hoc Network among Android smart devices." In Green Computing and Internet of Things (ICGCIoT), 2015 International Conference on, pp. 1322-1327. IEEE, 2015. DOI: https://doi.org/10.1109/ICGCIoT.2015.7380671

[23] Alam, Tanweer, and Mohammed Aljohani. "An approach to secure communication in mobile ad-hoc networks of Android devices." In 2015 International Conference on Intelligent Informatics and Biomedical Sciences (ICIIBMS), pp. 371-375. IEEE, 2015. DOI: https://doi.org/10.1109/iciibms.2015.7439466

[24] Aljohani, Mohammed, and Tanweer Alam. "An algorithm for accessing traffic database using wireless technologies." In Computational Intelligence and Computing Research (ICCIC), 2015 IEEE International Conference on, pp. 1-4. IEEE, 2015. DOI: https://doi.org/10.1109/iccic.2015.7435818

[25] Alam, Tanweer, and Mohammed Aljohani. "Design a new middleware for communication in ad hoc network of android smart devices." In Proceedings of the Second International Conference on Information and Communication Technology for Competitive Strategies, p. 38. ACM, 2016. DOI: https://doi.org/10.1145/2905055.2905244

[26] Bai, Yan, Lingsheng Yao, Tao Wei, Fei Tian, Dong-Yan Jin, Lijuan Chen, and Meiyun Wang. "Presumed asymptomatic carrier transmission of COVID-19." Jama 323, no. 14 (2020): 1406-1407. 